VOL. 50 (1994) [167-176]

\title{
CONVERGENCE OF \\ RELAXED CHAOTIC PARALLEL ITERATIVE METHODS
}

\author{
Peter E. Kloeden and Dong-Jin Yuan
}

Sufficient conditions involving uniform multisplittings are established for the convergence of relaxed and $A O R$ versions of asynchronous or chaotic parallel iterative methods for solving a large scale nonsingular system of linear equations $A x=b$.

\section{INTRODUCTION}

Parallel multisplitting iterative methods for solving a large system of linear equations

$$
A x=b,
$$

where $A \in \mathbb{R}^{n \times n}$, take two basic forms, synchronous when all of the processors wait until they are updated with the results of the current iteration before they begin with the next iteration or asynchronous when they act more or less independently of each other, using possibly delayed iterative values of the output of the other processors in computing their next iterate. In view of the potential time saving inherent in them, asynchronous iterative methods, or chaotic as they are often called, have attracted much attention since the early paper of Chazan and Miranker [2] introduced them in the context of point iterative schemes. Naturally, their convergence is of crucial interest and a number of convergence results have been obtained. Since relaxation of standard iterative methods often accelerates their convergence, the relaxation of asynchronous parallel methods may offer further time saving advantages provided convergence is assured. In this paper we investigate the convergence of two relaxed and three AOR methods which are based on methods of Elsner and his coworkers $[1,3,4]$ and of Su and Zhu [5] whose notation will be used in what follows.

Received 15th November, 1993.

This work was partially supported by the ARC grant A89132609.

Copyright Clearance Centre, Inc. Serial-fee code: 0004-9729/94 \$A2.00+0.00. 


\section{NOTATION}

Inequalities of vectors and matrices will be interpreted componentwise; in particular for $x=\left(x_{1}, \ldots, x_{n}\right) \in \mathbb{R}^{n}$ and $A=\left(a_{i j}\right) \in \mathbb{R}^{n \times n}$ by $x \geqslant 0$ we mean that $x_{i} \geqslant 0$ for $i=1, \ldots, n$ and by $A \geqslant 0$ that $a_{i j} \geqslant 0$ for $i, j=1, \ldots, n$, in which case we say that $x$ and $A$ are nonnegative.

For a matrix $A=\left(a_{i j}\right) \in \mathbb{R}^{n \times n}$, the absolute value matrix is $|A|=\left(\left|a_{i j}\right|\right)$ while the comparison matrix is $\langle A\rangle=\left(\langle a\rangle_{i j}\right)$ where

$$
\langle a\rangle_{i j}=\left\{\begin{array}{lll}
\left|a_{i j}\right| & \text { if } & i=j \\
-\left|a_{i j}\right| & \text { if } & i \neq j
\end{array}\right.
$$

A matrix $A=\left(a_{i j}\right) \in \mathbb{R}^{n \times n}$ is an $M$-matrix if it is nonsingular with $A^{-1} \geqslant 0$ and $a_{i j} \leqslant 0$ for all $i \neq j$. It is a $H$-matrix if $\langle A\rangle$ is an $M$-matrix, and an $L$-matrix if $a_{i i}>0$ for $i=1, \ldots, n$ and $a_{i j} \leqslant 0$ for all $i \neq j$.

A splitting of a matrix $A=\left(a_{i j}\right) \in \mathbb{R}^{n \times n}$ is a pair of matrices $M, N \in \mathbb{R}^{n \times n}$ with $\operatorname{det}(M) \neq 0$ such that $A=M-N$. It is called a nonegative splitting if $M^{-1} N \geqslant 0$ and an $M$-splitting if $M$ is an $M$-matrix and $N \geqslant 0$. For any $k \geqslant 2$ a multisplitting of $A \in \mathbb{R}^{n \times n}$ is a collection of $k$ triples $\left(M_{l}, N_{l}, E_{l}\right)$ of $n \times n$ real matrices, $l=1, \ldots, k$, for which each $E_{l}$ is nonnegative diagonal, each $M_{l}$ is invertible and the equalities

$$
A=M_{l}-N_{l}
$$

for $l=1, \ldots, k$ and

$$
\sum_{l=1}^{k} E_{l}=I_{n}
$$

are satisfied. A multisplitting $\left(M_{l}, N_{l}, E_{l}\right), l=1, \ldots, k$ is nonegative if the matrices $M_{l}, N_{l}$ for $l=1, \ldots, k$ are a nonnegative splittings of $A$ and uniform if there exist a scalar $\sigma \in(0,1)$ and a vector $\bar{x}>0$ such that

$$
M_{l}^{-1} N_{l} \bar{x} \leqslant \sigma \bar{x} \quad \text { for } \quad l=1, \ldots, k .
$$

Various conditions on the matrices $A, M_{l}$ and $N_{l}$ ensuring the uniformity of the multisplitting can be found in [1], for example, if $A$ is an $M$-matrix and the $M_{l}, N_{l}$ are $M$-splittings of $A$.

Finally, a sequence of sets $\left\{P_{i}\right\}$ with $P_{i} \subseteq\{1, \ldots, k\}$ is admissible if every integer $1, \ldots, k$ appears infinitely often in the $P_{i}$, while such an admissible sequence is regulated if there exists a positive integer $T$ such that each of the integers $1, \ldots, k$ appears at least once in any $T$ consecutive sets of the sequence. 
Using this notation we can now describe two models of parallel iteration from [1] for a given multisplitting of the coefficient matrix $A$. The first, model $\mathrm{A}$, is relatively straight forward with the form

$$
x^{i+1}=\sum_{l=1}^{k} E_{l} F_{l}^{\mu_{l i}}\left(x^{i}\right)
$$

for $i=0,1, \ldots$, where the $\mu_{l i} \geqslant 1$ are given integers and $F_{l}^{\mu_{l i}}$ is the $\mu_{l i}$-th composition of the affine mapping $F_{l}$ defined by

$$
F_{l}=M_{l}^{-1}\left(N_{l} x+b\right) .
$$

Here each processor in a parallel machine can carry out a varying number of iterations locally until a mutual phase time is reached when all processors are ready to contribute to the global iteration. The second, more complicated model B has

$$
x^{i+r_{i}}=\left(I_{n}-E_{j_{i}}\right) x^{i+r_{i}-1}+E_{j_{i}} F_{j_{i}}\left(z^{i}\right),
$$

for $i=-k+1, \ldots,-1,0,1, \ldots$, where $z^{i}=\left(\left(x_{1}^{i+r(1, i)}\right)^{\top}, \ldots,\left(x_{n}^{i+r(n, i)}\right)^{\top}\right)^{\top}$, the sequence $\left\{j_{i}\right\}$ is admissible and regulated for some positive integer $T, 0 \leqslant r(i, j) \leqslant$ $r_{i}-1 \leqslant T$ for $j=1, \ldots, n$, and $x^{-k+1}=\cdots x^{-1}=x^{0}$. Each processor here can update the global approximation or retrieve components of the global approximation from a central processor at any time provided no two processors do so at the same instant.

Both of the above models have been generalised by Su and Zhu [5] to allow for two or more processors to communicate with the central processor at the same time instant, in which case

$$
x^{i+r_{i}}=\left(I_{n}-\sum_{l \in P_{i}} E_{l}\right) x^{i+r_{i}-1}+\sum_{l \in P_{i}} E_{l} F_{l}^{\mu_{l i}}\left(z^{i}\right)
$$

where the $P_{i} \subseteq\{1, \ldots, k\}$ are admissible and regulated.

\section{RELAXED CHAOTIC ITERATIONS}

Here we consider two relaxed versions of the above parallel iteration models. The first, based on equation (5), uses a relaxation parameter $\omega$ in the same way as in a relaxation of the Jacobi method. It is

$$
x^{i+1}=\omega \sum_{l=1}^{k} E_{l} F_{l}^{\mu_{l i}}\left(x^{i}\right)+(1-\omega) x^{i},
$$


for $i=01, \ldots$ with $\omega>0$, and obviously reduces to (5) when $\omega=1$. The second is based on (8) and takes the form

$$
x^{i+1}=\left(I_{n}-\omega \sum_{l \in P_{i}} E_{l}\right) x^{i}+\omega \sum_{l \in P_{i}} E_{l} F_{l}^{\mu_{l i}}\left(w^{i-r_{i}+1}\right)
$$

for $i=0,1, \ldots$ with $\omega>0$, where $w^{i-r_{i}+1}=\left(\left(x_{1}^{i-r(1, i)}\right)^{\top}, \ldots,\left(x_{n}^{i-r(n, i)}\right)^{\top}\right)^{\top}$ and $P_{i} \subseteq\{1, \ldots, k\}$.

For both of these relaxed methods we have convergence under similar conditions.

Theorem 1. Suppose that $A \in \mathbb{R}^{n \times n}$ is invertible and $\left(M_{l}, N_{l}, E_{l}\right), l=1, \ldots, k$, is a nonegative uniform multisplitting of $A$. Then the relaxed parallel iterative method (9) converges to $x=A^{-1} b$ for any initial value $x^{0} \in \mathbb{R}^{n}$ when the relaxation parameter $\omega$ satisifies

$$
0<\omega<\frac{2}{1+\sigma}
$$

for the $\sigma$ in (4).

PROOF: To prove the theorem it suffices to show that the monotonic norms [1] of $n \times n$ real matrices $H_{i}$ defined by

$$
H_{i}=\omega \sum_{l=1}^{k} E_{l}\left(M_{l}^{-1} N_{l}\right)^{\mu_{l i}}+(1-\omega) I_{n}
$$

satisfy $\left\|H_{i}\right\|_{\bar{x}} \leqslant \beta$ for all $i$ and some $\beta \in(0,1)$, where $\bar{x}$ is from (4). Let $\beta=$ $\sigma \omega+|1-\omega|$, so $\beta<1$ for all $\omega$ satisfying (11). Post-multiplying the matrix $H_{i}$ by the vector $\bar{x}>0$ in (4), it follows from inequality (4) and the nonnegativity of the $E_{l}$ and the $M_{l}^{-1} N_{l}$ that

$$
\begin{aligned}
\left|H_{i} \bar{x}\right| & \leqslant \omega \sum_{l=1}^{k} E_{l}\left|M_{l}^{-1} N_{l}\right|^{\mu_{l i}} \bar{x}+|1-\omega| \bar{x} \\
& \leqslant \omega \sum_{l=1}^{k} E_{l}\left|M_{l}^{-1} N_{l}\right| \bar{x}+|1-\omega| \bar{x} \leqslant \omega \sigma \bar{x}+|1-\omega| \bar{x}=\beta \bar{x} .
\end{aligned}
$$

Thus we have $\left\|H_{i}\right\|_{\bar{x}} \leqslant \beta$ and convergence follows as in [1].

TheOREM 2. Suppose that $A \in \mathbb{R}^{n \times n}$ is invertible, that $\left(M_{l}, N_{l}, E_{l}\right), l=$ $1, \ldots, k$, is a nonegative uniform multisplitting of $A$, and that the index sequence 
$\left\{P_{i}\right\}$ is admissible and regulated. Then the relaxed parallel iterative method (10) converges to $x=A^{-1} b$ for any initial value $x^{0} \in \mathbb{R}^{n}$ when the relaxation parameter $\omega$ satisifies (11).

Proof: Let $x^{*}$ be the unique solution of $A x^{*}=b, \bar{x}$ the vector in the uniformity inequality (4) and $T \geqslant 2$ the integer in the regulatedness of the admissible index sequence $\left\{P_{i}\right\}$. Define $n T$-dimensional column vectors by

$X^{i}=\left(\left(x^{i}\right)^{\top}, \ldots,\left(x^{i-T+1}\right)^{\top}\right)^{\top}, \quad X^{*}=\left(\left(x^{*}\right)^{\top}, \ldots,\left(x^{*}\right)^{\top}\right)^{\top}, \quad \bar{X}=\left(\bar{x}^{\top}, \ldots, \bar{x}^{\top}\right)^{\top}$

and $n \times n T$ matrices $Z^{l i}=\left(Z_{1}^{l i}, \ldots, Z_{T}^{l i}\right)$ with components 0 or 1 such that $\sum_{s=1}^{T} Z_{s}^{l i}=I_{n}$ for $l=1, \ldots, k$ and $i=0,1, \ldots$ Then

$$
X^{*}-X^{i+1}=H_{i}\left(X^{*}-X^{i}\right)
$$

where

$$
H_{i}=\left[\begin{array}{lllll}
H_{i 1} & H_{i 2} & \ldots & H_{i T-1} & H_{i T} \\
I_{n} & 0 & \ldots & 0 & 0 \\
0 & I_{n} & \ldots & 0 & 0 \\
\ldots \ldots & \ldots \ldots \ldots \ldots \ldots \ldots \ldots \\
0 & 0 & \ldots & I_{n} & 0
\end{array}\right]
$$

with

$$
H_{i 1}=\left(I_{n}-\omega \sum_{l \in P_{i}} E_{l}\right)+\omega \sum_{l \in P_{i}} E_{l}\left(M_{l}^{-1} N_{l}\right)^{\mu_{l i}} Z_{1}^{l i}
$$

and

$$
H_{i s}=\omega \sum_{l \in P_{i}} E_{l}\left(M_{l}^{-1} N_{l}\right)^{\mu_{l i}} Z_{s}^{l i}
$$

for $s=2, \ldots, T$. Hence

$$
X^{*}-X^{i+2 T}=H_{i+2 T-1} H_{i+2 T-2} \cdots H_{i+1}\left(X^{*}-X^{i+1}\right)
$$

and since $H_{i} \geqslant 0$ for all $i$ to verify that $\left|X^{*}-X^{i}\right| \rightarrow 0$ as $i \rightarrow \infty$ it suffices to show that there exists a constant $\alpha \in[0,1)$ independent of $i$ such that the monotonic norm

$$
\left\|H_{i+2 T-1} H_{i+2 T-2} \cdots H_{i+1}\right\|_{\bar{X}} \leqslant \alpha
$$

for all $i$.

Define $\alpha=|1-\omega(1-\sigma) \delta|$ where $\sigma$ is from inequality (4) and

$$
\delta=\min _{\substack{j=1, \ldots, n \\ i=1,2, \ldots}}\left\{\sum_{l \in P_{i}}\left(E_{l}\right)_{j j}: \sum_{l \in P_{i}}\left(E_{l}\right)_{j j}>0\right\} \geqslant \min _{\substack{j=1, \ldots, n \\ l=1, \ldots, k}}\left\{\left(E_{l}\right)_{j j}:\left(E_{l}\right)_{j j}>0\right\},
$$


so $\delta \in(0,1]$. Hence $\alpha<1$ for $\omega$ satisfying (11).

Finally define $\bar{X}_{t}=\left(\left(\bar{x}_{t}^{1}\right)^{\top}, \ldots,\left(\bar{x}_{t}^{T}\right)^{\top}\right)^{\top}=H_{i+t} H_{i+t-1} \cdots H_{i+1} \bar{X}$ for $1 \leqslant t \leqslant$ $2 T-1$. From the uniformity inequality (4) and the definition of the $H_{t}$ it follows that $\bar{x}_{t}^{s} \leqslant \bar{x}$ for $1 \leqslant s \leqslant T$ since $H_{t} \bar{X} \leqslant \bar{X}$ for all $t \geqslant 1$. Hence for each $t \geqslant 1$

$$
\bar{x}_{t}^{1}=\left(I_{n}-\omega \sum_{l \in P_{q}} E_{l}\right) \bar{x}_{t-1}^{1}+\omega\left(\sum_{l \in P_{q}} E_{l}\left(M_{l}^{-1} N_{l}\right)^{\mu_{l i}} Z^{l i}\right) \bar{X}_{t-1}
$$

so by the properties of the multisplitting

$$
\begin{aligned}
\bar{x}_{t}^{1} & \leqslant\left(I_{n}-\omega \sum_{l \in P_{q}} E_{l}\right) \bar{x}+\omega\left(\sum_{l \in P_{q}} E_{l}\left(M_{l}^{-1} N_{l}\right)^{\mu_{l i}} Z^{l i}\right) \bar{X} \\
& =\left(I_{n}-\omega \sum_{l \in P_{q}} E_{l}\right) \bar{x}+\omega \sum_{l \in P_{q}} E_{l}\left(M_{l}^{-1} N_{l}\right)^{\mu_{l i}} \bar{x} \\
& \leqslant\left(I_{n}-\omega \sum_{l \in P_{q}} E_{l}\right) \bar{x}+\omega \sigma \sum_{l \in P_{q}} E_{l} \bar{x} \\
& \leqslant \bar{x}-\omega(1-\sigma) \sum_{l \in P_{q}} E_{l} \bar{x} .
\end{aligned}
$$

Now if $\sum_{l \in P_{i}}\left(E_{l}\right)_{j j}=0$ for some $j=1, \ldots, n$, then it follows from (13) that $\left(\bar{x}_{t}^{1}\right)_{j}=$ $\left(\bar{x}_{t-1}^{1}\right)_{j}$. On the other hand if $\sum_{l \in P_{i}}\left(E_{l}\right)_{j j}>0$, then from (14) we have

$$
\left(\bar{x}_{t}^{1}\right)_{j}=\left|\left(\bar{x}_{t}^{1}\right)_{j}\right| \leqslant|1-\omega(1-\sigma) \delta| \bar{x}_{j}=\alpha \bar{x}_{j} .
$$

Since the admissible sequence of index sets $\left\{P_{i}\right\}$ is regulated, each integer $1, \ldots, k$ must appear at least once in any segment $\left\{P_{i}, \ldots, P_{i+t}\right\}$ if $t \geqslant T$ and hence (15) holds for each $j=1, \ldots, n$ if $t \geqslant T$. Also from the definition of the $H_{t}$ it is clear that $\bar{x}_{2 T-1}^{s}=\bar{x}_{2 T-s-1}^{1}$ for $s=1, \ldots, T-1$. Hence (12) holds and the proof is complete.

\section{AOR CHAOTIC ITERATIONS}

Let $A \in \mathbb{R}^{n \times n}$ be nonsingular such that $D=\operatorname{diag} A$ is also nonsingular. For $l=1, \ldots, k$ let $L_{l} \in \mathbb{R}^{n \times n}$ be strictly lower triangular, define $U_{l}=D-L_{l}-A$ (which need not be upper triangular) and let $\left(D-L_{l}, U_{l}, E_{l}\right), l=1, \ldots, k$, be a multisplitting of $A$. Finally define

$$
F_{l}(r, \omega, x)=\left(D-r L_{l}\right)^{-1}\left\{\left[(1-\omega) D+(\omega-r) L_{l}+\omega U_{l}\right] x+\omega b\right\}
$$


for $r \geqslant 0, \omega>0, x \in \mathbb{R}^{n}$ and $l=1, \ldots, k$.

We consider three AOR versions of the above parallel iterative methods here. The first, based on (5), is

$$
x^{i+1}=\sum_{l=1}^{k} E_{l} F_{l}^{\mu_{l i}}\left(r, \omega, x^{i}\right),
$$

to which a positive relaxation parameter $\beta>0$ is introduced to yield the second,

$$
x^{i+1}=\beta \sum_{l=1}^{k} E_{l} F_{l}^{\mu_{l i}}\left(r, \omega, x^{i}\right)+(1-\beta) x^{i},
$$

while the final AOR method is the relaxed method (10) with $w^{i-r_{i}+1}$ replaced by $x^{i-r_{i}+1}$, that is

$$
x^{i+1}=\left(I_{n}-\omega \sum_{l \in P_{i}} E_{l}\right) x^{i}+\omega \sum_{l \in P_{i}} E_{l} F_{l}^{\mu_{l i}}\left(r, \omega, x^{i-r_{i}+1}\right) .
$$

As before, the $\mu_{l i}$ here are positive integers.

THEOREM 3. Suppose that $A \in \mathbb{R}^{n \times n}$ is an invertible $H$-matrix with multisplitting $\left(D-L_{l}, U_{l}, E_{l}\right), l=1, \ldots, k$, such that

(i) $D=\operatorname{diag} A, L_{l}$ is strictly lower triangular and $U_{l}=D-L_{l}-A$,

(ii) $\langle A\rangle=|D|-\left|L_{l}\right|-\left|U_{l}\right|$

for each $l=1, \ldots, k$. Then the AOR parallel iterative method (16) converges to $x=A^{-1} b$ for any initial value $x^{0} \in \mathbb{R}^{n}$ when the parameters $r$ and $\omega$ satisfy

$$
0 \leqslant r<\omega \text { and } 0<\omega<\frac{2}{1+\rho^{*}}
$$

where $\rho^{*}$ is the spectral radius of the matrix $|D|^{-1}(|D|-\langle A\rangle)$.

ProOF: As before it suffices to find a constant $\alpha \in(0,1)$ which is independent of $i$ and an appropriate vector $\bar{x} \in \mathbb{R}^{n}$ such that the monotonic norm $\left\|H(r, \omega)_{i}\right\|_{\bar{x}} \leqslant \alpha$ for all $i \geqslant 1$ where

$$
H(r, \omega)_{i}=\sum_{l=1}^{k} E_{l}\left\{\left(D-r L_{l}\right)^{-1}\left[(1-\omega) D+(\omega-r) L_{l}+\omega U_{l}\right]\right\}^{\mu_{l i}} .
$$

Now $A$ is an invertible $H$-matrix and $L_{l}$ is strictly lower triangular, so $\left\langle D-r L_{l}\right\rangle$ is an $L$-matrix and $\left\langle D-r L_{l}\right\rangle^{-1}=\left(|D|-r\left|L_{l}\right|\right)^{-1}>0$. Hence $D-r L_{l}$ is an invertible $H$-matrix and the matrix inequality

$$
\left|\left(D-r L_{l}\right)^{-1}\right| \leqslant\left\langle D-r L_{l}\right\rangle^{-1}=\left(|D|-r\left|L_{l}\right|\right)^{-1}
$$


holds, from which it follows that

$$
\begin{aligned}
\left|\left(D-r L_{l}\right)^{-1}\left[(1-\omega) D+(\omega-r) L_{l}+\omega U_{l}\right]\right| & \\
& \leqslant\left\langle D-r L_{l}\right\rangle^{-1}\left|(1-\omega) D+(\omega-r) L_{l}+\omega U_{l}\right| .
\end{aligned}
$$

Define

$$
M_{l}(r)=\left(|D|-r\left|L_{l}\right|\right)=\left\langle D-r L_{l}\right\rangle
$$

If $0 \leqslant r<\omega \leqslant 1$ define

$$
N_{l}^{(1)}(r, \omega)=(1-\omega)|D|+(\omega-r)\left|L_{l}\right|+\omega\left|U_{l}\right|
$$

and note that

$$
\left|(1-\omega) D+(\omega-r) L_{l}+\omega U_{l}\right| \leqslant N_{l}^{(1)}(r, \omega)
$$

and

$$
M_{l}(r)-N_{l}^{(1)}(r, \omega)=\omega\langle A\rangle .
$$

Since $M_{l}(r)$ is an $M$-matrix and $N_{l}^{(1)}(r, \omega) \geqslant 0$, the matrices $M_{l}(r), N_{l}^{(1)}(r, \omega)$ are an $M$-splitting of $\omega\langle A\rangle$, which is also an $M$-matrix, so $\left(M_{l}(r), N_{l}^{(1)}(r, \omega), E_{l}\right), l=$ $1, \ldots, k$, is a uniform multisplitting of $\omega\langle A\rangle$, with an $\bar{x}_{1}=\bar{x}_{1}(r, \omega)>0$ and an $\alpha_{1}=\alpha_{1}(r, \omega) \in(0,1)$ such that

$$
M_{l}(r)^{-1} N_{l}^{(1)}(r, \omega) \bar{x}_{1} \leqslant \alpha_{1} \bar{x}_{1}
$$

for $l=1, \ldots, k$. Hence

$$
\left|H(r, \omega)_{i} \bar{x}_{1}\right| \leqslant \sum_{l=1}^{k} E_{l}\left\{M_{l}(r)^{-1} N_{l}^{(1)}(r, \omega)\right\}^{\mu_{l, i}} \bar{x}_{1} \leqslant \sum_{l=1}^{k} E_{l} \alpha_{1} \bar{x}_{1} \leqslant \alpha_{1} \bar{x}_{1}
$$

On the other hand, if $0 \leqslant r<\omega$ and $1<\omega<2 /\left(1+\rho^{*}\right)$ define

$$
N_{l}^{(2)}(r, \omega)=(\omega-1)|D|+(\omega-r)\left|L_{l}\right|+\omega\left|U_{l}\right|
$$

and note that

$$
\left|(1-\omega) D+(\omega-r) L_{l}+\omega U_{l}\right| \leqslant N_{l}^{(2)}(r, \omega)
$$

and

$$
M_{l}(r)-N_{l}^{(1)}(r, \omega)=(2-\omega)|D|-\omega|B| .
$$

Since $M_{l}(r)$ is an $M$-matrix and $N_{l}^{(2)}(r, \omega) \geqslant 0$, the matrices $M_{l}(r), N_{l}^{(2)}(r, \omega)$ are an $M$-splitting of the matrix $(2-\omega)|D|-\omega|B|$, which is an $M$-matrix for $1<\omega<$ 
$2 /\left(1+\rho^{*}\right)$ by [6, Theorem 7.2]. In fact, $\left(M_{l}(r), N_{l}^{(1)}(r, \omega), E_{l}\right), l=1, \ldots, k$, is a uniform multisplitting of $(2-\omega)|D|-\omega|B|$, with an $\bar{x}_{2}=\bar{x}_{2}(r, \omega)>0$ and an $\alpha_{2}=\alpha_{2}(r, \omega) \in(0,1)$ such that

$$
M_{l}(r)^{-1} N_{l}^{(2)}(r, \omega) \bar{x}_{2} \leqslant \alpha_{2} \bar{x}_{2}
$$

for $l=1, \ldots, k$. Hence

$$
\left|H(r, \omega)_{i} \bar{x}_{2}\right| \leqslant \sum_{l=1}^{k} E_{l}\left\{M_{l}(r)^{-1} N_{l}^{(2)}(r, \omega)\right\}^{\mu_{l i}} \bar{x}_{2} \leqslant \sum_{l=1}^{k} E_{l} \alpha_{2} \bar{x}_{2} \leqslant \alpha_{2} \bar{x}_{2} .
$$

Inequalities (22) and (24) give the desired result.

In the statement and proof of the next theorem the quantities $\bar{x}_{1}, \alpha_{1}$ and $\bar{x}_{2}, \alpha_{2}$ are as in (21) and (23), respectively.

THEOREM 4. Suppose that the assumptions of Theorem 3 hold. Then the AOR parallel iterative method (17) converges to $x=A^{-1} b$ for any initial value $x^{0} \in \mathbb{R}^{n}$ when the parameters $r$ and $\omega$ satisfy inequalities (19) and

$$
0<\beta<\beta^{*}(r, \omega)
$$

where $\beta^{*}(r, \omega)=2 /\left(1+\alpha_{1}(r, \omega)\right)$ if $0<\omega \leqslant 1$ and $\beta^{*}(r, \omega)=2 /\left(1+\alpha_{2}(r, \omega)\right)$ if $1<\omega<\rho^{*}$.

Proof: Here too it suffices to find a constant $\lambda \in(0,1)$ which is independent of $i$ and an appropriate vector $\bar{x} \in \mathbb{R}^{n}$ such that the monotonic norm $\left\|H(r, \omega, \beta)_{i}\right\|_{\bar{x}} \leqslant \lambda$ for all $i \geqslant 1$ where

$$
H(r, \omega, \beta)_{i}=\beta H(r, \omega)_{i}+(1-\beta) I_{n}
$$

with $H(r, \omega)_{i}$ given by $(20)$.

Define $\lambda_{j}=|1-\beta|+\beta \alpha_{j}$ for $j=1$ and 2 .

Then $\lambda_{1} \in(0,1)$ for $0 \leqslant r<\omega \leqslant 1$ and $0<\beta<2 /\left(1+\alpha_{1}(r, \omega)\right)$, so

$$
\left|H(r, \omega, \beta)_{i} \bar{x}_{1}\right| \leqslant \beta \alpha_{1} \bar{x}_{1}+|1-\beta| \bar{x}_{1}=\lambda_{1} \bar{x}_{1} .
$$

On the other hand, $\lambda_{2} \in(0,1)$ for $0 \leqslant r<\omega, 1 \leqslant \omega<2 /\left(1+\rho^{*}\right)$ and $0<\beta<$ $2 /\left(1+\alpha_{2}(r, \omega)\right)$, so

$$
\left|H(r, \omega, \beta)_{i} \bar{x}_{2}\right| \leqslant \beta \alpha_{2} \bar{x}_{2}+|1-\beta| \bar{x}_{2}=\lambda_{2} \bar{x}_{2} .
$$

These give the desired bound on the matrix norm.

The proof of the our final result follows from those of Theorems 2 and 3.

THEOREM 5. Suppose that the assumptions of Theorem 3 hold and that the index sequence $\left\{P_{i}\right\}$ is admissible and regulated. Then the AOR parallel iterative method (18) converges to $x=A^{-1} b$ for any initial value $x^{0} \in \mathbb{R}^{n}$ when the parameters $r$ and $\omega$ satisfy inequalities (19). 


\section{References}

[1] R. Bru, L. Elsner and M. Neumann, 'Models of parallel chaotic iterative methods', Linear Algebra Appl. 103 (1988), 175-192.

[2] D. Chazan and W. Miranker, 'Chaotic relaxation', Linear Algebra Appl. 2 (1969), 199-222.

[3] L. Elsner, M. Neumann and B. Vemmer, 'The effect of the number of processors on the convergence of the parallel block Jacobi method', Linear Algebra Appl. 154/156 (1991), 311-330.

[4] L. Elsner and M. Neumann, 'Monotone sequences and rates of convergence of asynchronous iterative methods', Linear Algebra Appl. 180 (1993), 17-33.

[5] Y. Su and S. Zhu, 'Models of parallel multisplitting chaotic iterations methods', (in Chinese), J. Fudan Univ. Natur. Sci. 30 (1991), 444-450.

[6] D.M. Young, Iterative solution of large linear systems (Academic Press, New York, 1971).

School of Computing and Mathematics

Deakin University

Geelong Vic 3217

Australia
Yangzhou Teachers' College

Yangzhou

Peoples Republic of China 